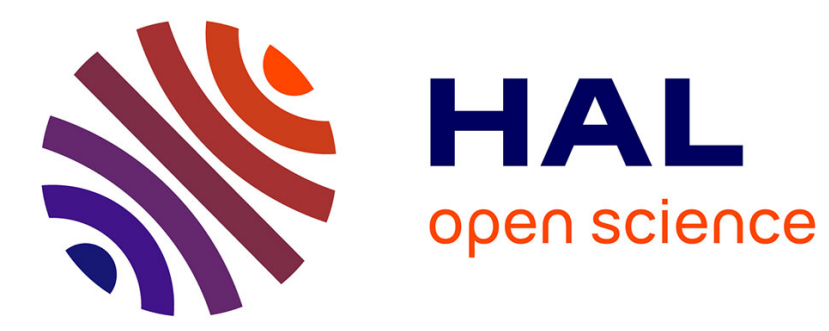

\title{
Sur une expérience de contrôle dynamique des connaissances en maîtrise de chimie physique
}

\author{
Roger F. F. Prat
}

\section{To cite this version:}

Roger F. F. Prat. Sur une expérience de contrôle dynamique des connaissances en maîtrise de chimie physique. Revue Française de Pédagogie, 1971, 14, pp.14. hal-00330593

\section{HAL Id: hal-00330593 \\ https://hal.science/hal-00330593}

Submitted on 15 Oct 2008

HAL is a multi-disciplinary open access archive for the deposit and dissemination of scientific research documents, whether they are published or not. The documents may come from teaching and research institutions in France or abroad, or from public or private research centers.
L'archive ouverte pluridisciplinaire HAL, est destinée au dépôt et à la diffusion de documents scientifiques de niveau recherche, publiés ou non, émanant des établissements d'enseignement et de recherche français ou étrangers, des laboratoires publics ou privés. 


\section{SUR UNE EXPERIENCE DE CONTROLE DYNAMIQUE DES CONNAISSANCES EN MAITRISE DE CHIMIE PHYSIQUE}

Depuis deux ans qu'est entamée l'analyse et la critique du système d'enseignement traditionnel, beaucoup de notions ont été popularisées, qui n'étaient jusque-là utilisées que par les spécialistes : notions de psychologie, de sociologie, d'économie politique, de pédagogie scientifique, etc. Certes le bouilionnement d'idées de 68 a vécu, mais le débat sur le rôle et la fonction de l'université est toujours ouvert. On prend un plaisir intellectuel de qualité à participer à ce débat. Cependant, certains esprits positifs constatèrent rapidement que tout cela était bel et bon, souhaitable et coherent, mais inapplicable, voire utopique, t en effet, faute de concevoir eux-mêmes des expériences pratiques destinées à vérifier et si besoin utjliser les constructions theoriques des chercheurs en psychopédagogie, ils s'empressèrent de revenir au statu quo ante, avec ses facteurs de tension, ses ambiguïtés et ses contradictions.

II n'est cependant pas vrai que la recherche psychopédagogique n'ait pas donné lieu à des applications concrètes. Bien au contraire : il existe actuellement de nombreuses écoles pilotes, tant publiques que privées, qui utilisent une technique d'enseignement et un matériel d'exercice entièrement basés sur les résultats d'observa. tions faites depuis environ un siècie sur des enfants à l'école. C'est qu'en effet, les connaissances à communi. quer a l'enfant de 7 ans ne sont pas tellement spéciali. sées qu'un chercheur en pédagogie ne puisse appliquer ce qu'il sait à la transmission de ces connaissances-là.

Mais on ne peut demander au psycho-pédagogue de savoir assez de biologie, assez de mécanique quantique ou assez de thermodynamique pour créer dans les facultés des sciences l'équivalent du materiel et des techniques qui font leurs preuves à l'âge de l'orthographe et du calcul.

S'il y a quelque chose dans la psycho-pédagogie qui nous concerne, c'est à nous, non spécialistes dans ce domaine, d'aller voir.

La moisson pourtant est décevante, et, à strictement parler, on revient bredouille. En effet, la psycho-pédagogie a actuellement pour objet surtout l'enfant à l'école primaire, et le jeune adolescent dans certains aspects particuliers. Le presque adulte ou l'adulte qu'est l'étudiant de faculté n'est pas encore objet de recherche. II nous a fallu faire une hypothèse de travail : certaines au moins des données psychologiques concernant l'enfant sont aussi, en permanence, présentes dans l'adulte ; c'est une hypothese cohérente avec une observation classique : lorsqu'un enfant n'a pas eu accès à tel matériel conçu pour une periode sensible déterminée, on observe que longtemps apres, alors que la période sensible est passée, il prendra beaucoup d'intérêt à ce matériel qui pourtant n'est plus de son åge : peut-on dire que les pères passionnés de trains électriques en ont fortement désiré un dans leur jeune temps? Ainsi, peut-être, certaines sources d'intérêt, brimées, déviées ou inexploitées, sontelles disponibles chez l'adulte.

Ainsi posée, la question n'est pas insoluble (les affirmations un peu sèches qui suivent ne doivent pas être prises au pied de la lettre; elles sont des affirmations statistiquement justifiées).

La plus grosse lacune dans l'éducation reçue pat notre étudiant statistique est celle qui concerne le développement de son esprit d'initiative. Depuis son plus jeune âge, tant dans sa famille qu'à l'école, it a, à proprement parler, reçu son éducation; on ne lui a que rarement laissé le temps de réaliser entièrement quelque chose, en le guidant seulement à sa demande, sans le cribler de conseils intempestifs. Les resultats de cette carence sont divers, et vont de l'adaptation à la révolte ouverte, en passant par la passivité en attendant de devenir grand. Mais alors, souvent, le pli est pris.

La deuxième lacune s'observe au sujet de l'aptitude à la communication : incapable de formuler problème ou solution, ou au contraire noyant son interlocuteur sous un verbiage dont il reconnaît l'herméticité chez son parte. 
naire, le jeune aduite manque de vocabulaire sur, faute d'avoir pu user des mots nouveaux en contrôlant qu'ils étaient bien employés à bon escient. On sait combien l'acquisition du vocabulaire est liee en fait à celle des connaissances. Malheureusement, face à l'organisation polarisante de l'enseignement, et même lorsque l'encadrement est suffisant en nombre, f'étudiant n'a pas non plus l'initiative pour parler. L'expérience montre que l'organisation actuelle des activités dirigées conduit à l'opposé de ce pourquoi elles étaient conçues: au lieu de séances animées où les étudiants dialoguent entre eux, faisant appel parfois à l'assistant qui est là, la passivité acquise des uns et le besoin de parler de l'assistant (car lui aussi en a été frustré, et il se venge) s'entendent pour transformer cela en cours.

La méthode classique d'enseignement cause d'autres dommages, plus subtils à discerner : ainsi, qui se soucie de rendre au contrôle des connaissances sa vertu éducative ? L'examen est devenu une épreuve redoutée que l'on passe pour le diplôme qu'il entraîne; pourtant, contrôler soj-même une construction mentale est une nécessité vitale pour l'enfant; le matériel psycho-pédagogique est toujours concu de sorte que chaque exercice avec le matériel puisse être sans ambiguïté vérifié. L'exemple le plus connu est celui de ces coupelles qui s'emboitent les unes dans les autres : la moindre erreur est immédiatement perceptible, mais n'entraîne pas de sanction extérieure, et l'enfant recommence plusieurs fois la manipulation de ce mémo matériel, jusqu'à ce qu'il soit sûr de ne plus se tromper. Si vous interrompez sa manipulation en lui déclarant que voici la dernière, que celle-lá compte, et qu'après on passe à autre chose, vous voyez le dégât. C'est pourtant de cette façon qu'intervient l'examen classique : nous interrompons brutalement le processus de maturation, plus ou moins long, selon les étudiants, et, encore une fois, voilà un travail inachevé.

II y a plus grave : il suffit que la discipline enseignée s'y prête tant soit peu, et l'examen devient un but en lui-même et change l'éclairage de la matière du cours, lequel se découpe en "sujets " de questions de cours, en "sujets" de problemes; nos esprits positifs discernent bien que, concrètement, c'est l'examen qu'il faut préparer; le terme de "bachotage " traduit l'activité de gavage accéléré qui précède l'épreuve : tant mieux si quelque chose d'autre qu'un diplôme demeure après l'examen. (Précisons que nous ne prenons pas ici position sur la notion de diplôme : nous avons affaire à des aduites, et, dans l'état actuel des choses, il est vital pour ces adultes d'en posséder. Cette motivation est réelle et actuelle, et nous observons seulement qu'elle est mal utilisée).

Laisser l'initiative du rythme de travail à l'enseigné, rendre plus probables les échanges horizontaux qui rodent le vocabulaire et les notions, utiliser la vertu édu- cative du contrôle des connaissances, tels sont les trois ingrédients intimement mélangés et indissociables de l'expérience que nous allons décrire, et dont le résultat espéré est précisément qu'il reste quelque chose d'autre qu'un diplôme après l'examen.

La matière du cours est considérée ici comme un donné qui est communiqué à un amphithéâtre composé d'étudiants en assez grand nombre (disons : plus de cinquante) pour que les traits de l'étudiant statistique ci-dessus soient observés. La façon dont le cours est enseignẻ peut être remise en cause, mais pas sa nature de donné, qui se concrétise par un certain volume de notes prises par chaque étudiant pour Jui-même. $\mathrm{Ce}$ volume augmente avec le temps, à une vitesse imposée le plus souvent, et elle-méme déterminée assez arbitrairement.

Dans notre expérience, la discipline enseignée est la mécanique quantique, c'est-à-dire une théorie physique bâtie pour expliquer certains phénomènes physiques surprenants, ef qui nécessite une mentalité hors du sens commun : par exemple l'abandon de la distinction entre l'objet mesuré et l'appareil de mesure. Le bagage mathématique minimum est assez important et les modèles résolus avec exactitude peu nombreux. Il y a assez de physique dans la mécanique quantique pour que le mathématicien ait de sérieuses difficultés d'assimilation, et assez de mathématiques pour dérouter un physico-chimiste. Pour nos étudiants, l'obstacle des mathématiques invariablement mobilisait toute leur attention, au détriment du contenu physique, qui est pourtant l'objet du cours.

Le matériel d'exercice et son mode d'emploi sont conçus pour l'assimilation complète du sujet, tant dans son esprit que dans ses méthodes; d'une part, il ne peut jouer le rôle tenu actuellement par le cours, d'autre part, i). perd la majeure partie de ses qualités s'il n'est pas utilisé collectivement, comme on le verra plus loin.

\section{Matériel}

Parce que théorie explicative de faits expérimentaux, une partie du matériel de mécanique quantique devrait donner accès concrètement au message physique contenu dans ces faits expérimentaux, convenablement analysés et symbolisés dans un matériel simple; transposé des coupelles citées plus haut, et ne comportant pour son utilisation aucune compétence technique à acquérir en surplus. Ce matériel reste à mettre au point. Nous avons do nous contenter de supposer que l'étudiant avait une connaissance livresque de ces faits.

L'autre partie du matériel doit soutenir l'étudiant dans son effort d'assimilation de la théorie abstraite et lui fournir de multiples occasions de mettre ses hypothèses à l'épreuve : nous avons rédigé une liste de questions se recouvrant partiellement, écrites sans préambule explicatif des notations, présentées sans ordre chronologique, avec 
parfois des données surabondantes, et couvrant tout l'ensemble du cours. Cette liste ne se distingue pas essentiellement d'une liste d'exercices de mécanique quantique; nous avons seulement cherché à éviter la répétition des schémas de solution, cette répétition étant mise à profit ailleurs.

\section{Modalitès}

La liste précédente a êté distribuée à l'amphithéâtre dès le début du cours, avec un texte explicatif dont nous extrayons les passages suivants :

" Chaque étudiant a la possibilité d'obtenir (...) une note de 0 à 20 qui interviendra avec le coefficient (...) dans la note officielle de fin d'année si.

« L'épreuve continue consiste d'une part à répondre par écrit, simultanément ou successivement a (trois) questions au moins choisies par l'étudiant (dans la liste) (...). Chaque étudiant peut rédiger plus de trois questions. S'il en rédige moins de trois pendant la période de validité (de la.liste), il est déclaré absent à l'épreuve continue des connaissances. Certaines des questions de la liste ci-dessus pourront en être rayées au cours de la période de validité, en particulier lorsque trop de candidats les choisissent (...) ".

* D'autre part, l'étudiant qui a remis une réponse rédigée se présentera à des séances (spéciales) où il pourra être interrogé sur le contenu de sa copie. L'interrogation orale est notée en elle-même (...) " .

"L'étudiant, par ce système, a la possibilité de doser son activité á sa guise, et de rédiger chaque réponse au mornent où il se juge le mieux préparé. En cas d'erreur de jugement, il peut se repêcher en répondant à plus de trois questions (...) $)$.

\section{Dynamique}

\section{Voyons à présent le fonctionnement.}

Qu'il possede ou non un cours polycopié, il est toujours rentable pour l'étudiant d'avoir dès le début un recueil d'exercices adaptés par hypothèse à sa formation antérieure. A la première lecture, les questions posées sont toutes « difficiles ", pour ne pas dire de l'hébreu ; réparties sans ordre, il faudra lire et relire la liste pour repérer ceux des exercices qui sont devenus * possibles " par les notions entendues au cours; éventuellement en discuter, ou, en entendre discuter. Pendant ce temps, les activités dirigées habituelles se sont poursuivies, puisqu'il entrait dans la nature de l'expérience de n'étudier que quelques points précis, toutes choses égales par ailleurs.

Puis, c'est là que commence le bénéfice de l'utilisation collective de la liste, arrivent les premières questions rédigées. Les motivations en sont diverses, dont par exemple l'idée (sans fondement) que les premiers auraient le choix des questions les plus « faciles", ou encore le sentiment que rendre une question $n^{\circ} 33$ imparfaite n'est pas un drame irréversible : on perd seulement la possibilité de rédiger à nouveau cette question $n^{\circ} 33$. De toutes façons, l'arrivée des premières copies montre que le groupe des étudiants commence à mûrir vis-à-vis du $n^{\circ} 33$. Les séances de contrôle oral se déroulent devant vingt-cinq étudiants. Le candidat interrogé sur la question $n^{\circ} 33$ qu'il a remise une semaine auparavant ne corrige pas cet exercice, if lui est proposé une autre question voisine de celle-là et assez souvent inspirée par telle difficulté qui transparaît dans sa rédaction : c'est le moment de répéter les schémas de solution, en variant la forme de la question subsidiaire. Les camarades présents sont eux aussi concernés par la question posée (1) qui fournit quantité de renseignements complémentaires : chacun apprend que le cours permet de résoudre la question 33 ; les réponses aux questions subsidiaires donnent des idées : on est aux aguets. Quand plusieurs questions sont simultanément abordées au tableau, le groupe de vingtcinq se partage spontanément en plus petits groupes qui s'intéressent plus à l'un des exercices qu'aux autres: travail en commun dans l'amphi fl'assistant est la pour dépanner) et épreuves solitaires au tableau. La différence avec une séance de travaux dirigés classique tient essentiellement à ce qu'icl l'étudiant choisit, et choisit l'exercice pour lequel il est le plus mûr parce qu'il a déjà réfléchi aux questions semblables de la liste. Aucune de ces séances spéciales n'a besoin d'être "obligatoire "; elles sont assidament fréquentées. La discussion d'exercices periphériques autour de la question $n^{\circ} 33$ familiarise avec les notions, les notations et le vocabulaire, sans faire perơre l'intérêt qu'il y a à la rédiger. Bien qu'il soit a priori possible de copier mot a mot une solution type, le cas ne s'est pas présenté; en tout état de cause, l'oral serait là pour discerner si l'étudiant a effectivement le niveau qu'il montre dans sa copie.

Bientôt s'établit dans l'amphi un " régime permanent " de discussions autour de plusieurs questions à la fois couvrant un éventail assez large, dont la gamme progresse au rythme des suppressions de questions en amont; le mécanisme par lequel un exercice sera officiellement corrigé, donc disparaîtra de la liste, a été dans la mesure du possible déclenché par l'amphi lui-même: nous avons attendu que se produise la croissance rapide (en une semaine parfois) du nombre de copies remises sur la question $n^{\circ} 33$, signe de la maturité du groupe pour ce sujet, pour la stopper. Cependant, afin de garder aux questions périphériques une certaine diversité, il est pré-

(1) au contraire d'un oral d'examen, où l'étudiant qui assiste a l'interogation d'un camarade sait qu'il a peu de chances d'avoir les mêmes questions. 
férable de ne pas laisser rédiger chaque exercice beaucoup plus d'une quinzaine de fois.

Plusieurs observations ont été faites, dont certaines demandent à être confirmées :

a) sur un questionnaire de quarante-sept exercices, seuls quatre d'entre-eux n'ont pas été choisis, parmi lesquels figure une question sur le début du cours. On peut penser a priori qu'il s'agit soit de questions hors sujet, soit mal rédigées ;

b) sur 102 étudiants, 81 d'entre-eux ont participé à ce type d'épreuve. (On notera qu'il faut de toutes facons prévoir pour les autres un examen complémentaire classique sous forme, ici, de problème à faire en trois heures) 370 copies ont été rendues, ce qui donne une moyenne de 4 à 5 copies par étudiant (pour trois obligatoires) ;

c) la maturation la plus rapide a été observée pour des questions spécifiquement quantiques, ayant trait aux propriétés du spin. La remarque tire son intérêt du fait que c'est lá précisément la partie du cours sur laquelle achoppent les débutants.

Nous aurions voulu connaitre le degré de maturité de l'amphi à l'époque où habituellement avait lieu un examen partiel classique, vers la mi-décembre. Malheureusement les modalités et les conditions de l'expérience venaient à peine d'être comprises; et les dernières méfiances levées, de sorte que peu de copies avajent été remises à ce moment. Quoi qu'il en soit :

d) la liberté d'étaler le contrôle a été pleinement utilisée, avec cependant une croissance plus vive du nombre des copies rendues chaque semaine aux environs de la fin du cours. Nous ne pouvons cependant interpréter cette dernière observation car les étudiants subissaient en même temps des examens partiels dans d'autres matières ;

e) plusieurs étudiants, isolés ou travaillant en groupe ont rendu plus de dix copies. Avant de passer l'oral, nous donnons connaissance à l'étudiant de sa note d'écrit, et il décide alors de la " défendre " ou au contraire l'abandonne. De ce fait, 345 copies (sur 370 remises) ont été contrôlées à l'oral ;

f) la majeure partie des notes définitives fécrit plus oral) se situe entre 10 et 18 , avec un maximum vers. 16 . Nous n'attribuons pas suffisamment de crédit aux valeurs prises par les notes pour conclure sur ce critère au succès de l'expérience. Nous tirons de cette observation seulement ceci : l'épreuve continue des connaissances produit un groupe plus homogène vis-à-vis de la matiere enseignée, puisqu'on observe un seul maximum accentué dans la courbe de distribution des notes, et non, comme souvent, plusieurs sous-maxima. Cette conclusion, à la réflexion, était prévisible : les étudiants qui n'ont jamais été * mârs * (il y en a une vingtaine) n'ont pas rendu de copies et ne figurent pas dans le graphique : confirmation est fournie par le résultat de l'examen complémentaire classique pour cette vingtaine.

\section{Résultat}

Nous avons volontairement omis de parler des conditions matérielles de l'expérience; peut-être sont-elles trop locales pour présenter de l'intérêt. Nous noterons seulement qu'avec un enseignant en moyenne pour vingtcinq étudiants, on peut envisager de remplacer le contrôle discret habituel par un tel contrôle véritablement continu, en conservant seulement en plus les activités dirigées de correction de problème et d'explication du cours.

Pour juger du succès ou de l'échec de l'épreuve continue, nous avons utilisé le résultat fourni par une épreuve classique indépendante que les étudiants du certificat ont subie alors que notre expérience était presque terminée. Soulignons qu'ici exceptionnellement l'examen classique a une valeur réelle de témoignage : en effet, il n'a pas eu lieu en cours de maturation, mais alors que le nombre et l'étendue des questions rédigées et contrōlées montraient concrètement le degré élevé atteint dans l'assimilation du cours au moment où il a été passé : l'examen pouvait avoir lieu, $80 \%$ de l'amphithéatre était prêt, sans bachotage.

Le résultat est résumé dans la courbe de distribution ci-dessous ; on y voit la séparation nette de l'amphithéătre en une petite minorité qui n'est pas encore au point, et un groupe homogène qui a assimilé les idées, mais a manipulé les équations avec plus ou moins de bonheur. Interrogé sur cette épreuve, l'étudiant statistique répond que l'examen partiel était facile, mais qu'il y avait beaucoup de calculs, ce qui est exactement le sens que nous venons de donner à la courbe.

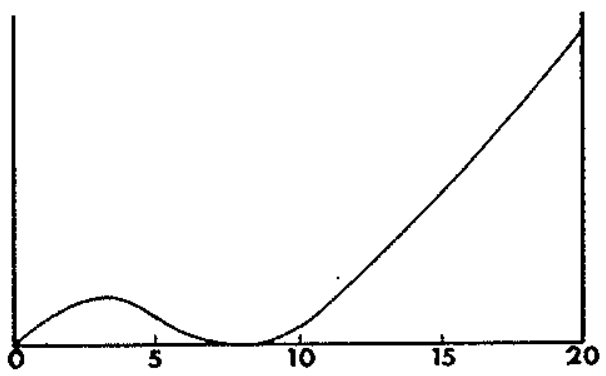

\section{Conclusion}

Un large champ de recherches peut être ouvert dans le domaine des applications de la psycho-pédagogie dans l'enseignement des sciences en faculté; il ne peut étre déblayé que par des enseignants-chercheurs en sciences. 
A partir d'une hypothèse de travail, nous avons conçu une expérience qui montre que l'acquisition des connaissances ne doit pas être séparée de l'étape "contrôle ", mais qu'il est primordial que celui-ci soit spontané et collectif. Dans la méthode expérimentée, chaque étudiant dose son activité a sa guise, mais est cependant stimulé de façon naturelle par l'ambiance de discussion qui s'établit dans l'amphithéâtre gråce à une utilisation judicieuse d'un matériel d'exercice. L'amphithéátre se transforme rapidement en un groupe où les informations circulent, où le vocabulaire se rode, où la pédagogie par les pairs déblaye le terrain et prépare l'intervention de l'assistant. Quand il se sent prêt, l'étudiant prend l'initiative de se faire contrôler. La méthode cependant ne nécessite pas davantage d'encadrement que la technique traditionnelle.

\section{Remerclements}

Cette expérience n'eût pas été possible sans la liberté que nous a donnée $M$. Rouault, professeur chargé du cours de mécanique quantique, de l'organiser; bien que nos précautions aient été prises en cas d'accidents de parcours, encore fallait-il franchir le pas qui sépare un accord intellectuel sur les principes, de la prise de responsabilités réelles, que $M$. Rouault a assumées.

Roger F. PRAT, maitre-assistant à la Faculté des Sciences d'Orsay 